\title{
Inductive Logic from the Viewpoint of Quantum Information
}

\author{
Vasil Penchev, vasildinev@gmail.com \\ Bulgarian Academy of Sciences: Institute of Philosophy and Sociology: \\ Dept. of Logical Systems and Models
}

\begin{abstract}
The resolving of the main problem of quantum mechanics about how a quantum leap and a smooth motion can be uniformly described resolves also the problem of how a distribution of reliable data and a sequence of deductive conclusions can be uniformly described by means of a relevant wave function " $\Psi_{\text {data" }}$.

Key words: entanglement, hypothetical reasoning interpretation of wave function, inductive and probabilistic conclusion
\end{abstract}

Any inductive conclusion is a hypothetical reasoning. Any finite set of absolutely reliable data is not sufficient for a deductive conclusion on their base. Thus any inductive conclusion suggests a hypothesis and respectively, its choice among all possible alternative hypotheses. That hypothesis complements the finite set of available reliable data so that it is able to imply a necessary conclusion.

There is a special case: a "zero" hypothesis of "Hypotheses non fingo". It means the hypothesis that the available data are not only a sufficient, but also necessary condition for the conclusion. As a corollary of its hypothetical essence, Popper's (1935) falsifiability can be interpreted as a principle stating that any available data cannot be ever a necessary condition for an ultimate conclusion. However, that hypothesis has the advantage to be the simplest one. Occam's razor remains just it. The principle of maximal entropy formalizes it quantitatively: One should choice that function approximating the available reliable data, the entropy of which is maximal.

Nevertheless earlier or later, it will be ever refused therefore being replaced by one of those "nonzero hypotheses" anyway consistent to any preliminary subset of reliable data. The non-zero hypotheses can be compared with each other about the probability for each of them to turn out the future zero and thus dominating hypothesis on the base of the data available only till now. Furthermore and quantitatively, any distribution of non-maximal entropy can be in interpreted as hidden factors or variables thinkable as a single one, which is just what generates the shift from the maximal entropy distribution.

The conception of quantum information was introduced in the theory of quantum information studying the phenomena of entanglement in quantum mechanics. The entanglement was theoretically forecast in the famous papers of Einstein, Podolsky, and Rosen (1935) and independently by Shrödinger (1935) deducing it from Hilbert space, the basic mathematical formalism of quantum mechanics. However, the former three demonstrated the forecast phenomenon as the proof of the alleged "incompleteness of quantum mechanics". John Bell (1964) deduced a sufficient condition as an experimentally verifiable criterion in order to distinguish classical from quantum correlation (entanglement). Aspect, Grangier, and Roger $(1981,1982)$ confirmed experimentally the existence of quantum correlations exceeding the upper limit of the possible classical correlations.

Quantum information generalizes information to infinite sets or transfinite ordinals. Both definitions of 'ordinal' (Cantor 1897; Neumann 1923) are applicable as the ordinals are small. The ordinal defined in Cantor - Russell (Whitehead, Russell any edition) generates a statistical ensemble while that in Neumann, a well-ordering. Both correspond one-to-one to a coherent state as the one and same quantity of quantum information containing in it. The theorems about the absence of hidden variables in quantum mechanics (Neumann 1932; Kochen, Specker 1968) demonstrate that the mathematical formalism of 
quantum mechanics implies that no well-ordering of any coherent state might exist before measurement. However, the same coherent state is transformed into a well-ordered series of results in time after measurement. In order to be equated the state before and after measurement, the well-ordering theorem equivalent to the axiom of choice is necessary. The unit of quantum information is a quantum bit (qubit), which can be interpreted as a generalization of a bit (an elementary choice) as to infinite series or sets: It is: $\alpha|0\rangle+\beta|1\rangle$ where $\alpha, \beta$ are complex numbers such that $|\alpha|^{2}+|\beta|^{2}=1$, and $|0\rangle,|1\rangle$ are any two orthonormal vectors (e.g. the orthonormal bases of any two subspaces) in any vector space (e.g. Hilbert space, Euclidean space, etc.). Thus Hilbert space underlying quantum mechanics is representable as the quantity of quantum information and any wave function, i.e. any state of any quantum system being a point in it can be seen as a value of that quantity.

The resolving of the main problem of quantum mechanics about how a quantum leap and a smooth motion can be uniformly described resolves also the problem of how a distribution of reliable data and a sequence of deductive conclusions can be uniformly described by means of a relevant wave function " $\Psi_{\text {data" }}$ ".

Furthermore, any "non-zero" hypothesis about some set of reliable data is equivalent to an entanglement to another wave function " $\Psi_{\text {hypothesis }}$ ". The entanglement in turn is equivalent to a new resultant wave function " $\Psi_{\text {hypothesis (data)", which can be interpreted both a distribution of non-maximal }}$ entropy and a reordered sequence of conclusions. For the latter to be a tautology, a modified set of axioms is necessary. The relation of the initial and modified set of axioms represents axiomatically the non-zero hypothesis in question.

Thus the method of quantum information allows of mapping between the distributions of nonmaximal entropy and the axiomatic systems expressing various "non-zero" hypotheses.

\section{References:}

Aspect, A., Grangier, R., Roger, G. (1981) “Experimental Tests of Realistic Local Theories via Bell's Theorem," Physical Review Letters 47 (7): 460-463.

Aspect, A., Grangier, R., Roger, G. (1982) "Experimental Realization of Einstein-Podolsky-Rosen-Bohm Gedanken Experiment: A New Violation of Bell's Inequalities," Physical Review Letters 49 (2): 91-94.

Bell, J. (1964) “On the Einstein - Podolsky - Rosen paradox,” Physics (New York) 1 (3): 195-200.

Cantor, G. (1897) "Beitrage zur Begrundung der transfiniten Mengenlehre (Zweiter Artikel)," Mathematische Annalen 49 (2): 207-246.

Einstein, A., Podolsky, B., Rosen, N. (1935) "Can Quantum-Mechanical Description of Physical Reality Be Considered Complete?” Physical Review 47 (10): 777-780.

Kochen, S., Specker, E. (1968) "The problem of hidden variables in quantum mechanics," Journal of Mathematics and Mechanics 17 (1): 59-87.

Neumann, J. von (1923) "Zur Einführung der trasfiniten Zahlen," Acta litterarum ac scientiarum Ragiae Universitatis Hungaricae Francisco-Josephinae, Sectio scientiarum mathematicarum 1 (4): 199-208.

Neumann, J. von (1932). Mathematische Grundlagen der Quantenmechanik. Berlin: Springer, pp. 167-173 (Chapter IV.2).

Popper, K. (1935) Logik der forschung: zur erkenntnistheorie der modernen naturwissenschaft. Wien: Springer.

Schrödinger, E. (1935) "Die gegenwärtige situation in der Quantenmechanik," Die Naturwissenschaften, 23 (48), 807-812; 23 (49), 823-828, 23 (50), 844-849.

Whitehead, A. N., Russell, B, (any edition) Principia Mathematica, Vol. 2(*153), Vol. 3(*251). 